\title{
members of commissions, boards, and committees'
}

\section{EXECUTIVE COMMITTEE ${ }^{2}$}

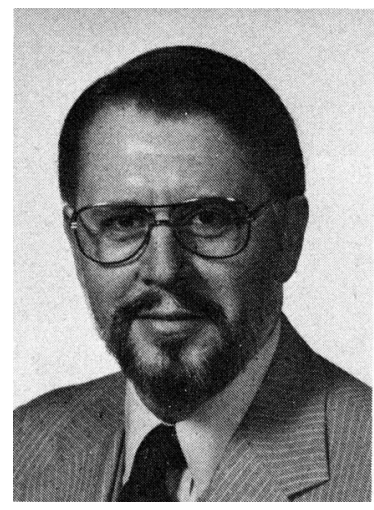

E. W. Bierly

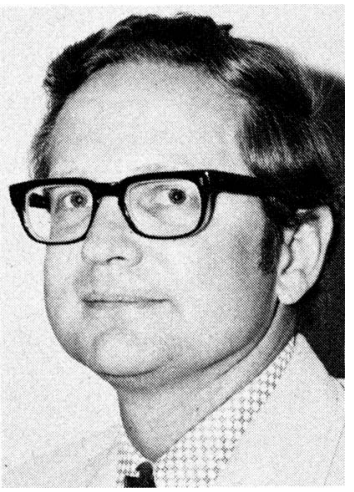

H. D. Orville

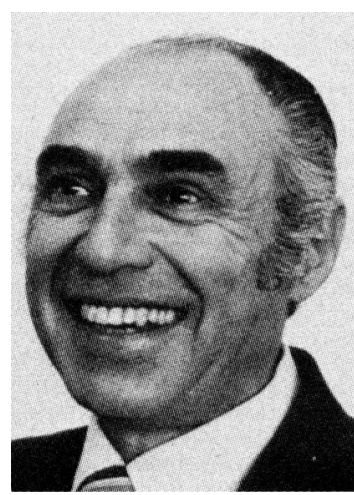

C. J. Murino

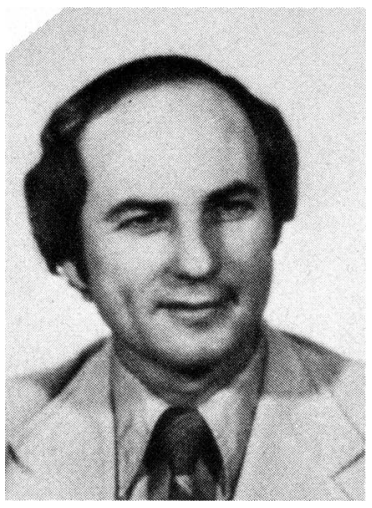

T. H. Vonder Haar

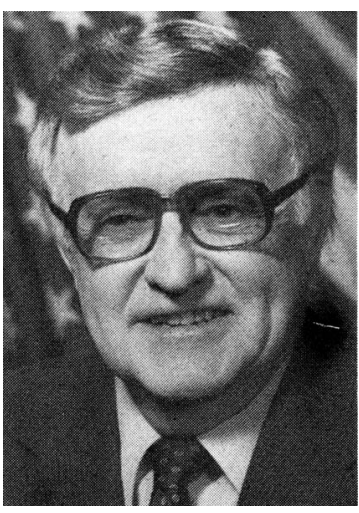

E. G. Droessler

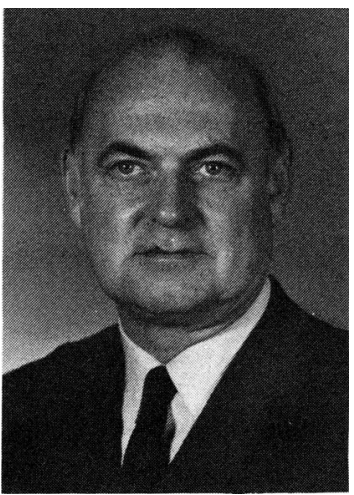

K. C. Spengler

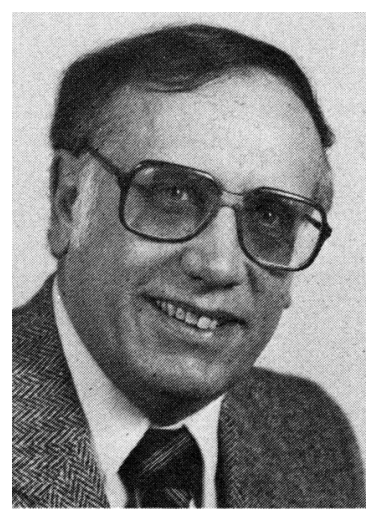

R. E. Hallgren

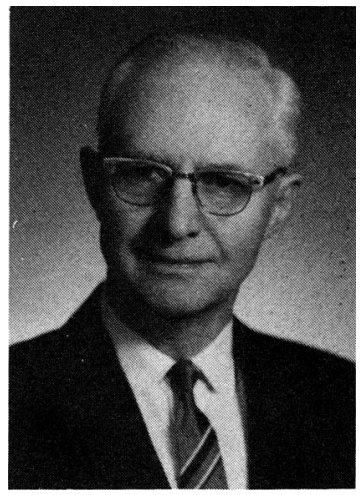

D. F. Landrigan

\section{COMMITTEES OF THE EXECUTIVE COMMITTEE}

\section{Awards}

Chairman: Dr. Chester W. Newton, National Center for Atmospheric Research, P.O. Box 3000, Boulder, CO 80307

Dr. Glenn R. Hilst, Electric Power Research Institute, P.O. Box 10412, Palo Alto, CA 94303 Prof. David D. Houghton, Dept. of Meteorology, University of Wisconsin-Madison, Madison, WI 53706

Prof. James J. O’Brien, Meteorology Annex, Florida State University, Tallahassee, FL 32306 Dr. James L. Rasmussen, Director, Office of Meteorology, National Weather Service, NOAA, 8060 13th St., Silver Spring, MD 20910

\footnotetext{
${ }^{1}$ The Executive Director is an ex officio member of all commissions, boards, and committees.

${ }^{2}$ Addresses listed on preceding page.
} 


\section{Nominating ${ }^{3}$}

\section{Admissions}

\section{Ethics}

\section{History of the Atmospheric Sciences}

\section{Investment $\mathbf{t}^{3,4}$}

Chairman: Prof. Robert G. Fleagle, Dept. of Atmospheric Sciences, University of Washington, Seattle, WA 98195

Dr. D. Ray Booker, President, Aeromet, Inc., P.O. Box 45447, Tulsa, OK 74145

Dr. Philip E. Merilees, CCCD-Atmospheric Environment Service, 4905 Dufferin St., Toronto, Ont., Canada M3H 5T4

Prof. Richard E. Orville, Dept. of Atmospheric Science, State University of New York at Albany, 1400 Washington Ave., Albany, NY 12222

Dr. Douglas H. Sargent, Director, Office of Systems Development, National Weather Service, NOAA, 8060 13th St., Silver Spring, MD 20910

Chairman: Valentine J. Descamps, U.S. Environmental Protection Agency, Region 1, J. F. Kennedy Federal Bldg., Boston, MA 02203

Owen R. Côté, Atmospheric Sciences Division, Air Force Geophysics Laboratory, Hanscom AFB, MA 01731

David B. Spiegler, The Mitre Corporation, P.O. Box 208, Bedford, MA 01730

John E. Wallace, President, Weather Services Corporation, 131A Great Rd., Bedford, MA 01730 John W. Wilson, Stone and Webster Engineering Corporation, P.O. Box 2325, Boston, MA 02107

Chairman: Richard G. Semonin, Assistant Chief for Administration and Research, Illinois State Water Survey, P.O. Box 5050, Station A, Champaign, IL 61820-9050

Keith J. Brown, North American Weather Consultants, 1141 E. 3900 S., A130, Salt Lake City, UT 84117

Dr. Neil L. Frank, Director, National Hurricane Center, NOAA, 1320 S. Dixie Highway, Coral Gables, FL 33146

Jerry D. Hill, Climate Assessment Technology, Inc., 11550 Fuqua, Suite 355, Houston, TX 77034

Charles F. Roberts, P.O. Box 639, New Market, VA 22844

Chairman: Dr. Ronald C. Taylor, Atmospheric Research Section, National Science Foundation, 1800 G St., N.W., Washington, DC 20550

Prof. Robert D. Bornstein, Dept. of Meteorology, San Jose State University, San Jose CA 95192 John H. Conover, 15 Nobel Rd., Dedham, MA 02026

J. Patrick Hughes, NESDIS/NOAA, E/ER3, FOB4, Rm. 330, Washington, DC 20233

Dr. Gisela Kutzbach, 1138 Shorewood Blvd., Madison, WI 53705

Prof. Helmut E. Landsberg, Institute of Physical Science and Technology, University of Maryland, College Park, MD 20742

Chairman: Dr. Werner A. Baum, Dean, College of Arts and Sciences, Florida State University, Tallahassee, FL 32306

Eugene Bollay, 411 Los Robles Ln., Santa Barbara, CA 93105

Prof. Charles L. Hosler, Dean, College of Earth and Mineral Sciences, Pennsylvania State University, 116 Deike Bldg., University Park, PA 16802

Dr. Thomas F. Malone, Unit 203, 5 Bishop Rd., W. Hartford, CT 06119

\section{COMMITTEES OF THE COUNCIL}

Fellows $^{3}$

Chairman: Peter R. Leavitt, Weather Services Corporation, 131A Great Rd., Bedford, MA 01730 Prof. James R. Holton, Dept. of Atmospheric Sciences, University of Washington, Seattle, WA 98195

Frederick P. Ostby, Jr., Director, National Severe Storms Forecast Center, NOAA, 601 E. 12th St., Kansas City, MO 64106

\footnotetext{
${ }^{3}$ The AMS President serves ex officio on this committee.

${ }^{4}$ The Chairman of the Planning Commission, the Executive Director, and the Secretary-Treasurer serve ex officio on this committee.
} 
Prof. Jesse J. Stephens, Dept. of Meteorology, Florida State University, Tallahassee, FL 32306 Prof. Warren S. Wooster, Institute for Marine Studies, University of Washington, Seattle, WA 98195

Sverdrup Gold Medal Chairman: Dr. Robert W. Stewart, Deputy Minister, Ministry of Universities, Science and Communications, Parliament Buildings, Room 109, Victoria, B.C., Canada V8V 1X4

Dr. Kirk Bryan, Jr., Geophysical Fluid Dynamics Laboratory, NOAA, Princeton University, P.O. Box 308, Princeton, NJ 08540

Dr. Jerome Namias, Scripps Institution of Oceanography, University of California, San Diego, La Jolla, CA 92093

Prof. Owen M. Phillips, Dept. of Earth and Planetary Sciences, Johns Hopkins University, Baltimore, MD 21218

Public Policy

Chairman: Dr. Eugene W. Bierly, Director, Div. of Atmospheric Sciences, National Science Foundation, 1800 G St., N.W., Washington, DC 20550

Dr. Clifford J. Murino, President, University Corporation for Atmospheric Research, P.O. Box 3000, Boulder, CO 80307

Dr. Earl G. Droessler, Assistant Secretary for Natural Resources, North Carolina Dept. of Natural Resources and Community Development, P.O. Box 27687, Raleigh, NC 27611

Dr. Richard E. Hallgren, Assistant Administrator for Weather Services, National Oceanic and Atmospheric Administration, 8060 13th St., Silver Spring, MD 20910

Prof. Robert G. Fleagle, Dept. of Atmospheric Sciences, University of Washington, Seattle, WA 98195

Dr. Joanne Simpson, Head, Severe Storms Branch, Goddard Laboratory for Atmospheric Sciences, Code 914, NASA, Greenbelt, MD 20771

\section{COMMISSION ON PROFESSIONAL AFFAIRS}

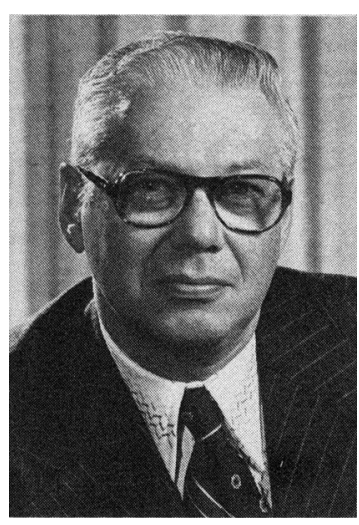

W. A. Baum

Board of Certified Consulting Meteorologists

Board of Broadcast Meteorology
Chairman: Prof. Werner A. Baum, Dean, College of Arts and Sciences, Florida State University, Tallahassee, FL 32306

Members: Chairmen of the constituent Boards

Chairman: Dr. George E. McVehil, P.O. Box 4480, Boulder, CO 80306

William H. Haggard, Climatological Consulting Corporation, Route 2, Box 372, Asheville, NC 28805

Dr. Walter A. Lyons, R*Scan Corporation, 511 11th Ave. S., Minneapolis, MN 55415

Dennis C. Perryman, Oceanroutes, Inc., 3260 Hillview Ave., Palo Alto, CA 94304

David B. Smith, Chas. T. Main, Inc., Prudential Center, Boston, MA 02199

Chairman: David P. Murray, ABC Entertainment, 7 W. 66th St., New York, NY 10023

Joel P. Bartlett, Jr., KPIX-TV 5, 855 Battery St., San Francisco, CA 94111

Dennis W. Feltgen, KSTP Weather Services, 3415 University Ave., St. Paul, MN 55414

Todd S. Glickman, WSI Corporation, 41 North Rd., Bedford, MA 01730

Thomas J. Mahoney, WFRV-TV 5, 1181 E. Mason St., Green Bay, WI 54305

Leonard M. Slesick, KVII-TV, Box 13000, Amarillo, TX 79101

Michael R. Smith, WeatherData, Inc., 833 N. Main St., Wichita, KS 67203 


\section{Board of Private Sector Meteorology}

Chairman: Timothy C. Spangler, WESTEC Services, 3211 5th Ave., San Diego, CA 92103 Jack G. Coblenz, Dames \& Moore, 4321 Directors Row, Houston, TX 77092

Richard T. Egami, Desert Research Institute, University of Nevada, P.O. Box 60220, Reno, NV 89506

Paul M. Fransioli, Black \& Veatch Consulting Engineers, P.O. Box 8405, Kansas City, MO 54114 Thomas F. Lavery, ERT, Inc., 696 Virginia Rd., Concord, MA 01742

Dr. Jerry Pell, Office of Coal Resource Management, Department of Energy, Washington, DC 20461

Board for Operational Government Meteorologists
Chairman: Dr. Kenneth C. Crawford, National Weather Service Forecast Office, NOAA, P.O. Box 59997, Oklahoma City, OK 73159

Christopher D. Hill, National Weather Service Forecast Office, 3905 Vista Ave., Boise, ID 83705 John A. Jannuzzi, National Weather Service Forecast Office, NOAA, 5420 N.E. Marine Dr., Portland, OR 97218

Jay S. Rosenthal, 1902 S. Parnell Ave., W. Los Angeles, CA 90025

Capt. Jesus B. Tupaz, USN, NSTL Station, Bay St. Louis, MS 39522

Eugene M. Weber, Air Force Global Weather Central (WF), Offutt AFB, NB 68113

\section{PUBLICATIONS COMMISSION}

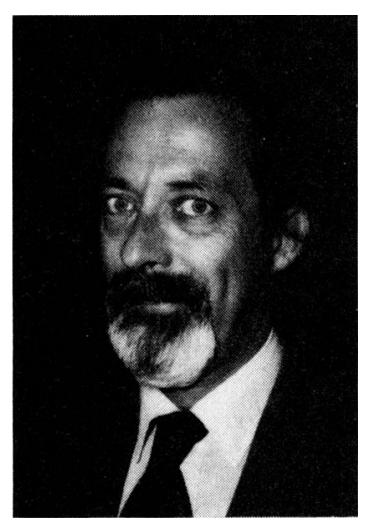

J. A. Dutton
Chairman: Prof. John A. Dutton, Dept. of Meteorology, Pennsylvania State University, University Park, PA 16802

Members: Editors-in-Chief of periodicals and serials

Members at Large:

Dr. Duncan C. Blanchard, Atmospheric Research Center, State University of New York-Albany, 1400 Washington Ave., Albany, NY 12222

Prof. Joshua Z. Holland, Dept. of Meteorology, University of Maryland, College Park, MD 20742

Dr. James R. Mahoney, Environmental Sciences Department, Bechtel Corporation, P.O. Box 3965, San Francisco, CA 94119

\section{Board of the Journal of the Atmospheric Sciences}

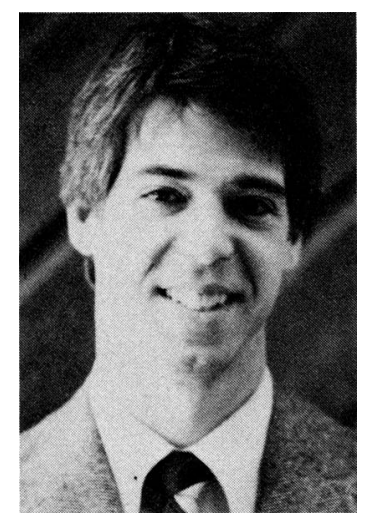

J. M. Fritsch

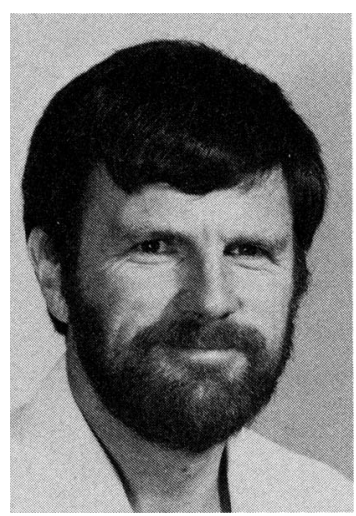

P. J. Webster
Co-Chief Editor: Prof. J. Michael Fritsch, Dept. of Meteorology, Pennsylvania State University, 503 Walker Bldg., University Park, PA 16802

Co-Chief Editor: Dr. Peter J. Webster, Dept. of Meteorology, Pennsylvania State University, 503 Walker Bldg., University Park, PA 16802 
JAS Editors:

Prof. Stephen K. Cox, Dept. of Atmospheric Sciences, Colorado State University, Ft. Collins, CO 80523

Prof. Conway B. Leovy, Dept. of Atmospheric Sciences, University of Washington, Seattle, WA 98195

Prof. Mankin Mak, Dept. of Atmospheric Sciences, University of Illinois, Urbana, IL 61801

Prof. Harold D. Orville, Dept. of Meteorology, South Dakota School of Mines and Technology, Rapid City, SD 57701

\section{JAS Associate Editors:}

Richard A. Anthes, National Center for Atmospheric Research

Donna Blake, Naval Ocean Research and Development Activity

Chih-Pei Chang, Naval Postgraduate School

Charles F. Chappell, National Oceanic and Atmospheric Administration

Barney J. Conrath, NASA/Goddard Space Flight Center

Robert E. Dickinson, National Center for Atmospheric Research

Kerry A. Emanuel, Massachusetts Institute of Technology

Marvin A. Geller, NASA/Goddard Space Flight Center

John E. Hart, University of Colorado

Dennis L. Hartmann, University of Washington

Isaac M. Held, GFDL/NOAA, Princeton University

James R. Holton, University of Washington

Brian J. Hoskins, University of Reading, U.K.

Robert A. Houze, Jr., University of Washington

Richard H. Johnson, Colorado State University

Eugenia Kalnay, NASA/Goddard Space Flight Center

Steven E. Koch, NASA/Goddard Space Flight Center

T. N. Krishnamurti, Florida State University

Colleen A. Leary, Texas Tech University

Jerry D. Mahlman, GFDL/NOAA, Princeton University

Robert A. McClatchey, Air Force Geophysics Laboratory

Alan Plumb, GFDL/NOAA, Princeton University

Hans R. Pruppacher, University of California-Los Angeles

V. Ramanathan, National Center for Atmospheric Research

Edward S. Sarachik, Harvard University

Susan Solomon, National Oceanic and Atmospheric Administration

Graeme L. Stephens, Division of Atmospheric Research, CSIRO, Australia

Duane E. Stevens, Colorado State University

John M. Wallace, University of Washington

John C. Wyngaard, National Center for Atmospheric Research

\section{Board of the Journal of Climate and Applied Meteorology}

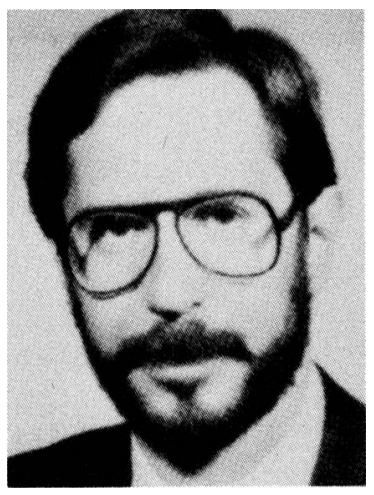

A. D. Hecht

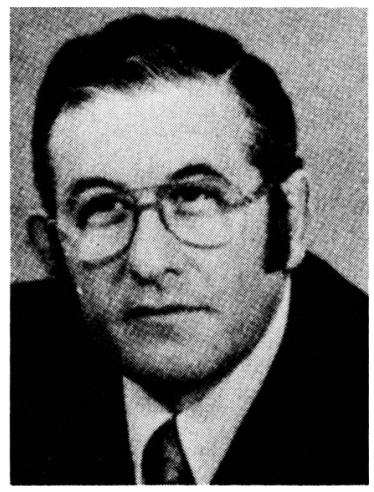

B. A. Silverman
Co-Chief Editor/Climate: Dr. Alan D. Hecht, National Climate Program Office, NOAA, 6010 Executive Blvd., Rockville, MD 20852

Co-Chief Editor/Applied Meteorology: Dr. Bernard A. Silverman, P.O. Box 2857, Littleton, CO 80161

\section{JCAM Editors:}

Dr. Kenneth H. Bergman, National Weather Service, NOAA, Washington, DC 20233

Richard E. Carbone, Field Observing Facility, National Center for Atmospheric Research, P.O. Box 3000, Boulder, CO 80307

Prof. William L. Smith, Cooperative Institute for Meteorological Satellite Studies, 1225 W. Dayton St., Madison, WI 53706 
JCAM Associate Editors:

Roger G. Barry, University of Colorado

William A. Cooper, University of Wyoming

Arnold Court, California State University, Northridge

Stephen K. Cox, Colorado State University

Robert J. Curran, NASA, Washington, D.C.

Richard J. Doviak, National Severe Storms Lab., NOAA

William P. Elliott, Air Resources Labs., NOAA

Henry E. Fleming, National Environmental Satellite Service, NOAA

Kenneth S. Gage, Environmental Research Labs., NOAA

Tzvi Gal-Chen, University of Oklahoma

W. Lawrence Gates, Oregon State University

Steven R. Hanna, ERT, Inc.

Christopher M. Hayden, NOAA/NESDIS/DL/SDA

Frederick B. House, Drexel University

Charles A. Knight, National Center for Atmospheric Research

Helmut E. Landsberg, University of Maryland

Thomas B. McKee, Colorado State University

Paul W. Mielke, Jr., Colorado State University

Eugene M. Rasmusson, Climate Analysis Center, NOAA/NWS

Norman J. Rosenberg, University of Nebraska

Robert S. Schemenauer, Atmospheric Environment Service, Canada

Robert J. Serafin, National Center for Atmospheric Research

Charles W. Stockton, University of Arizona

\section{Board of the Journal of Physical Oceanography}

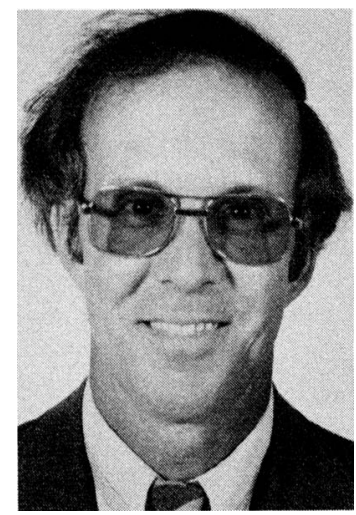

R. L. Haney

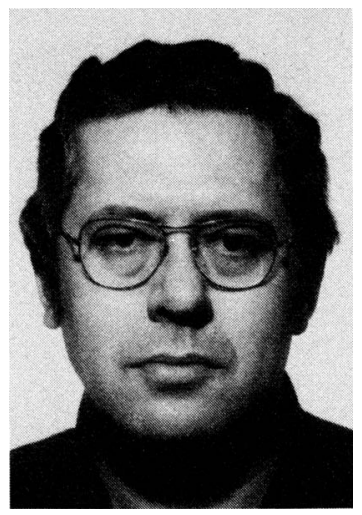

B. A. Warren
Co-Editor: Dr. Robert L. Haney, Dept. of Meteorology, Naval Postgraduate School, Monterey, CA 93943

Co-Editor: Dr. Bruce A. Warren, Woods Hole Oceanographic Institution, Woods Hole, MA 02543
JPO Associate Editors:

Robert C. Beardsley, Woods Hole Oceanographic Institution Melbourne G. Briscoe, Woods Hole Oceanographic Institution Roland W. Garwood, Naval Postgraduate School Terrence M. Joyce, Woods Hole Oceanographic Institution Lorenz C. Magaard, University of Hawaii

James C. McWilliams, National Center for Atmospheric Research

Lawrence A. Mysak, University of British Columbia Pearn P. Niiler, Scripps Institution of Oceanography James J. O’Brien, Florida State University Peter B. Rhines, Woods Hole Oceanographic Institution 


\section{Board of the Monthly Weather Review}

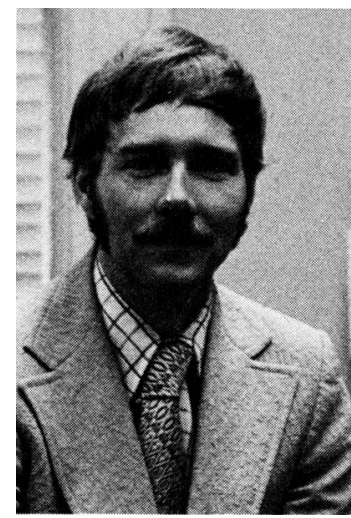

R. A. Pielke

Chief Editor: Dr. Roger A. Pielke, Dept. of Atmospheric Science, Colorado State University, Ft. Collins, CO 80523

\section{MRW Editors:}

Dr. William M. Frank, Dept. of Meteorology, Pennsylvania State University, University Park, PA 16802

Dr. Robert Maddox, ERL/NOAA, Boulder, CO 80303

Dr. Kevin E. Trenberth, National Center for Atmospheric Research, P.O. Box 3000, Boulder, CO 80307

\section{MRW Associate Editors:}

Richard A. Anthes, NCAR, Boulder, Colo.

David A. Barber, North Carolina State University

Kenneth C. Crawford, NOAA/NWS Forecast Office, Oklahoma City, Okla.

Roger Daley, NCAR, Boulder, Colo.

Charles A. Doswell, NOAA/ERL, Boulder, Colo.

Michael Garstang, Simpson Weather Associates, Inc., Charlottesville, Va.

Roland A. Madden, NCAR, Boulder, Colo.

Paul W. Mielke, Jr., Colorado State University

Rudolph W. Preisendorfer, NOAA/PMEL, Seattle, Wash.

Eugene M. Rasmusson, Climate Analysis Center, Washington, D.C.

Richard J. Reed, University of Washington

Richard D. Rosen, Atmospheric and Environmental Research, Inc., Cambridge, Mass.

Thomas Schlatter, NOAA/ERL, Boulder, Colo.

Roderick A. Scofield, NOAA/NESDIS, Washington, D.C.

Lloyd J. Shapiro, AOML/NOAA, Miami, Fla.

Joanne Simpson, Goddard Space Flight Center, Greenbelt, Md.

Graeme Stevens, CSIRO, Australia

Thomas T. Warner, Pennsylvania State University

Edward A. Zipser, NCAR, Boulder, Colo.

\section{Board of the Journal of Atmospheric and Oceanic Technology}

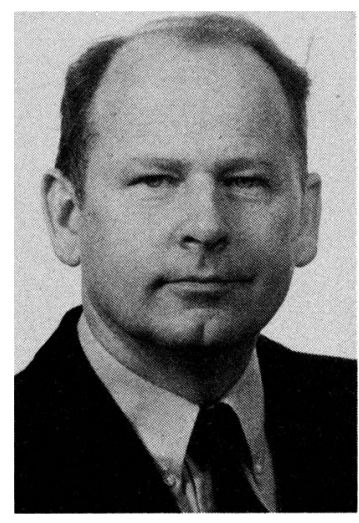

H. T. Rossby

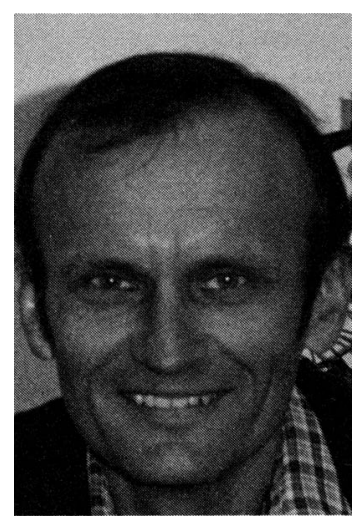

R. J. Serafin
Co-Editor: Prof. H. Thomas Rossby, Graduate School of Oceanography, University of Rhode Island, Kingston, RI 02881

Co-Editor: Dr. Robert J. Serafin, National Center for Atmospheric Research, P.O. Box 3000, Boulder, CO 80307 


\section{JAOT Associate Editors:}

William Bandeen, NASA/Goddard Space Flight Center Henri Berteaux, WHOI/Department of Ocean Engineering Fred Brock, NCAR/Field Observing Facility

Otis Brown, Rosenstiel School of Marine and Atmospheric Sciences, University of Miami Douglas Caldwell, School of Oceanography, Oregon State University

William A. Cooper, University of Wyoming

Robert W. Corell, Marine Programs, University of New Hampshire

Russ Davis, SIO/University of California-San Diego

Richard Doviak, NOAA/National Severe Storms Laboratory

James Dye, NCAR/Convective Storms Division

Arthur Few, Rice University

George Isaac, Atmospheric Environment Service, Downsview, Ontario, Canada

William G. Large, NCAR/Oceanography Division

Alvin Morris, Ambient Analysis, Inc. Boulder, Colorado

Douglas Sargeant, NOAA/NWS/Systems Development Office

Robert Spindel, WHOI/Department of Ocean Engineering

Ray Weiss, SIO/University of California-San Diego

Frank Wentz, Remote Sensing Systems, Inc., Sausalito, California

\section{Board of Meteorological Monographs}

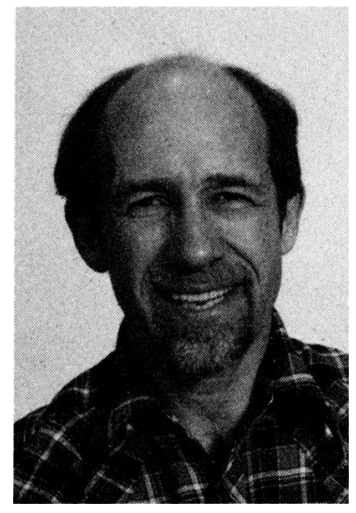

W. Blumen

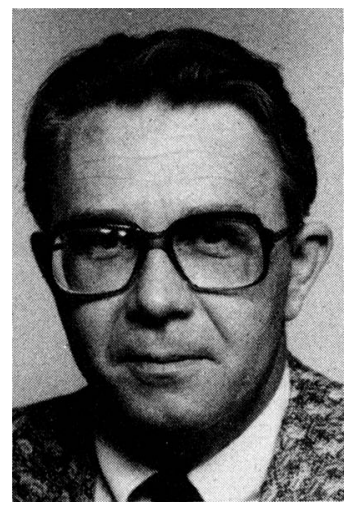

R. C. Taylor

Editor: Prof. William Blumen, Department of Astrophysical, Planetary, and Atmospheric Sciences, University of Colorado, P.O. Box 391, Boulder, CO 80309

\section{Monograph Associate Editors:}

Dr. Robert C. Beardsley, Woods Hole Oceanographic Institution, Woods Hole, MA 02543

Prof. James R. Holton, Dept. of Atmospheric Sciences, University of Washington, Seattle, WA 98195

Prof. Richard E. Orville, Dept. of Atmospheric Science, State University of New York at Albany, Albany, NY 12222

Prof. Jesse J. Stephens, Dept. of Meteorology, Florida State University, Tallahassee, FL 32306

Historical Monograph Series Editor:

Dr. Ronald C. Taylor, Meteorology Program, Division of Atmospheric Sciences, National Science Foundation, Washington, DC 20550 


\section{Bulletin of the American \\ Meteorological Society}

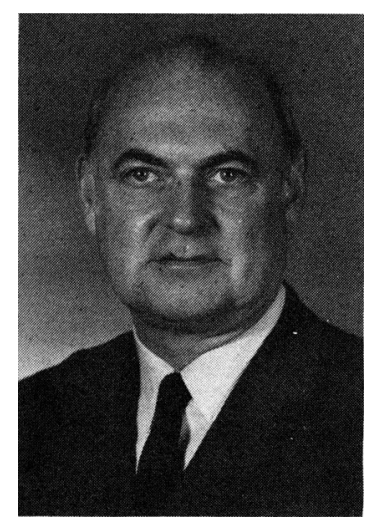

K. C. Spengler
Editor: Dr. Kenneth C. Spengler, Executive Director, American Meteorological Society, 45 Beacon St., Boston, Mass. 02108

\section{Board of Meteorological and Geoastrophysical Abstracts}

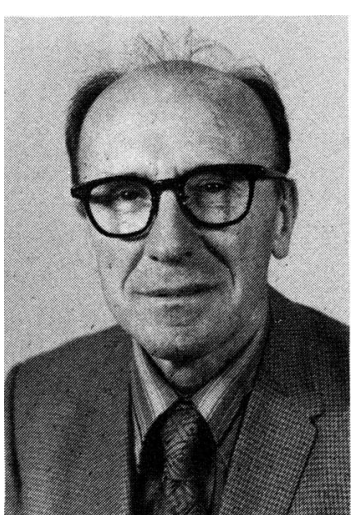

M. Rigby
Editor: Malcolm Rigby, 5816 22nd St. N., Arlington, Va. 22205

\section{SCIENTIFIC AND TECHNOLOGICAL ACTIVITIES COMMISSION ${ }^{7}$}

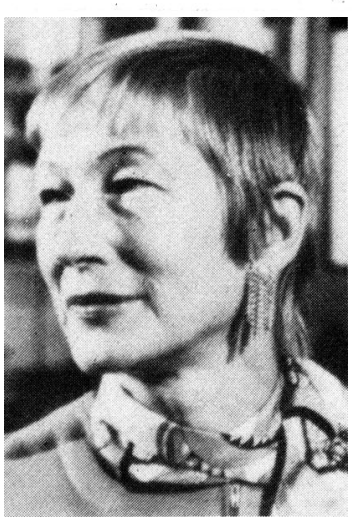

J. Simpson

\section{Agricultural and Forest Meteorology}

Chairman: Dr. Joanne Simpson, Head, Severe Storms Branch, Code 914, Goddard Laboratory for Atmospheric Sciences, NASA, Greenbelt, MD 20771

Members: Chairmen of the Scientific and Technological Activities Committees

Chairman: Dr. William A. Dugas, Blacklands Research Center, P.O. Box 748, Temple, TX 76501 Prof. D. M. Brown, Dept. of Resource Science, University of Guelph, Guelph, Ontario, Canada N1G 2W1

Dr. R. William Furman, Rocky Mountain Forest and Range Experiment Station, 240 W. Prospect, Ft. Collins, CO 80526

Prof. Lloyd W. Gay, Dept. of Hydrology, University of Arizona, Tucson, AZ 85721

Dr. Jerry L. Hatfield, USDA-ARS, Texas Tech University, P.O. Box 4170, Lubbock, TX 79409

Prof. Lawrence E. Hipps, Dept. of Soil Science and Biometeorology, Utah State University, Logan, UT 84322

Dr. Raymond P. Motha, World Food and Ágriculture Outlook Br., USDA, Rm. 5133-So. Bldg., Washington, DC 20250

Clyde A. O'Dell, National Weather Service, NOAA, 3905 Vista Ave., Boise, ID 83705

Prof. Peter H. Schuepp, Box 223, Mac Donald Campus, McGill University, St. Anne de Bellevue, Quebec, Canada H9X 1 C0

Dr. S. Verma, Center for Agricultural Climatology and Meteorology, University of Nebraska, Lincoln, NE 68583

Chairman: Prof. Marie E. Sanderson, Dept. of Geography, University of Windsor, Windsor, Ontario, Canada N9B 3P4

Prof. Arnold Court, Climatology-Dept. of Geography, California State University, Northridge, CA 91330

\footnotetext{
${ }^{7}$ The Chairman of STAC and the Executive Director of AMS are ex officio members of all STAC committees.
} 


\section{Atmospheric Chemistry and Radioactivity}

\section{Atmospheric \\ Electricity}

Prof. Robert F. Dale, Dept. of Agronomy, Purdue University, W. Lafayette, IN 47907

Dr. Nathaniel Guttman, Chief, Statistical Climatology Branch, National Climatic Ctr., EDIS/ NOAA/Department of Commerce, Federal Bldg., Asheville, NC 28801

Dr. James D. Laver, W/NMC 53, U.S. Department of Commerce, WWB, Rm. 210, Washington, DC 20233

Dr. J. Owen Rhea, Electronics Techniques, Inc., 471 1/2 Maidre, Auburn, CA 95603

Prof. William E. Riebsame, Dept. of Geography, University of Wyoming, Laramie, WY 82071

Dr. Ward R. Seguin, Chief, Local Applications Branch, Technical Development Laboratory, NWS/NOAA, Gramax Building, Silver Spring, MD 20910

Hilda J. Snelling, USAF/ETAC/ECE, Scott AFB, IL 62225

Dr. Wayne M. Wendland, Illinois Institute of Natural Resources, Illinois State Water Survey, 605 East Springfield Ave., P.O. Box 5050, Station A, Champaign, IL 61820

Chairman: Dr. Arland H. Johannes, Dept. of Chemical and Environmental Engineering, Rensselaer Polytechnic Institute, Troy, NY 12181

Dr. Leonard A. Barrie, Atmospheric Environmental Service, 4905 Dufferin St., Downsview, Ontario, Canada M3H 5T4

Dr. Ralph J. Cicerone, National Center for Atmospheric Research, P.O. Box 3000, Boulder, CO 80307

Prof. James Galloway, Dept. of Environmental Science, University of Virginia, Charlottesville, VA 22903

Prof. Volker A. Mohnen, Atmospheric Sciences Research Center, Earth Science Bldg.-Rm. 324, 1400 Washington Ave., State University of New York at Albany, Albany, NY 12222

Prof. Ronald G. Prinn, Dept. of Meteorology and Oceanography, Massachusetts Institute of Technology, Cambridge, MA 02139

Prof. Joseph M. Prospero, Rosenstiel School of Marine and Atmospheric Sciences, University of Miami, 4600 Rickenbacker Causeway, Miami, FL 33149

Prof. Kenneth A. Rahn, Graduate School of Oceanography, University of Rhode Island, Narragansett, RI 02882-1197

Dr. Jack D. Shannon, Radiological and Environmental Research Division, Argonne National Laboratory, 9700 Cass Ave., Argonne, IL 60439

Dr. Douglas M. Whelpdale, AES, ARQT, Atmospheric Environmental Service, 4905 Dufferin, Downsview, Ontario, Canada M3H 5T4

Dr. George T. Wolff, General Motors Research Laboratory, Warren, MI 48090

Chairman: Dr. W. David Rust, National Severe Storms Laboratory, NOAA/Department of Commerce, 1313 Halley Circle, Norman, OK 73069

Dr. Hugh Christian, Mail Code ED43, Atmospheric Physics Br., Atmospheric Sciences Div., NASA/Marshall Space Flight Center, Marshall Space Flight Center, AL 35812

Prof. John Hallett, Desert Research Institute, P.O. Box 60220, Reno, NV 89509

Prof. Craig O. Hayenga, Dept. of Physics, New Mexico Institute of Mining and Technology, Socorro, NM 87801

Dr. Edwin Kessler, III, Director, National Severe Storms Laboratory, Department of Commerce/NOAA, Norman, OK 73069

Prof. Philip Krider, Institute of Atmospheric Physics, Bldg. \#81, University of Arizona, Tucson, AZ 85721

Michael W. Maier, Lightning Location and Protection, Inc., 1001 South Euclid Ave., Tucson, AZ 85719

Prof. Richard E. Orville, Dept. of Atmospheric Sciences, State University of New York at Albany, 1400 Washington Ave., Albany, NY 12222

Dr. E. J. Stansbury, Vice-Principal for Planning, McGill University, Montreal, Quebec, Canada H3A 2TS

Chairman: Robert N. Swanson, Pacific Gas and Electric Co., 215 Market St., Rm. 458, San Francisco, CA 94106 
Atmospheric and Oceanic Waves and Stability

\section{Atmospheric Turbulence and Diffusion}

Dr. Fred V. Brock, National Center for Atmospheric Research, Field Observing Facility, P.O. Box 3000, Boulder, CO 80307

Dr. Richard L. Coulter, Argonne National Laboratory, Bldg. 181, 9700 S. Cass Ave., Argonne, IL 60439

Dr. Constantinos B. Emmanuel, Director, Research Flight Center, NOAA/Department of Commerce, P.O. Box 520197, Miami, FL 33152

Dr. Peter L. Finkelstein, Environmental Protection Agency/EMSL, Environmental Research Ctr. Annex, Mail Drop 56, Research Triangle Pk., NC 27711

Dr. Rayford P. Hosker, Jr., NOAA/ATDL, P.O. Box E, Oak Ridge, TN 37830

Dr. Robert A. Kropfli, Department of Commerce, WPL/ERL/NOAA, 325 Broadway, Boulder, CO 80303

Dr. Donald H. Lenschow, National Center for Atmospheric Research, P.O. Box 3000, Boulder, CO 80307

Dr. James D. McTaggart-Cowan, Director, Environmental Studies Revolving Funds Manager, Canada Oil and Gas Lands Administration, 355 River Road, Ottawa, Ontario, Canada K1A OE4

Chairman: Prof. William Blumen, APAS, University of Colorado, P.O. Box 391, Boulder, CO 80309

Prof. Albert I. Barcilon, GSDI, Keen Bldg.-Rm. 18, Florida State University, Tallahassee, FL 32306

Dr. Kenneth S. Gage, Atmospheric Dynamics, Aeronomy Laboratory, NOAA/ERL, Department of Commerce, 325 Broadway, Boulder, CO 80303

Dr. Dale B. Haidvogel, National Center for Atmospheric Research, P.O. Box 3000, Boulder, CO 80307

Prof. Lawrence A. Mysak, University of British Columbia, Dept. of Oceanography, 6270 University Blvd., Vancouver, B.C., Canada V6T 1 W5

Dr. Eugenia Kalnay, NASA/Goddard Space Flight Center, Modeling and Simulation Facility, Code 911, Greenbelt, MD 20771

Dr. Breck Owens, Woods Hole Oceanographic Institution, Woods Hole, MA 02543

Dr. D. B. Rao, NASA/Goddard Space Flight Center, Oceans and Ice Br.-Code 912, Greenbelt, MD 20771

Prof. David J. Raymond, Department of Physics, New Mexico Institute of Mining and Technology, Socorro, NM 87811

Chairman: Dr. Jeffrey C. Weil, Martin Marietta Corporation, 1450 S. Rolling Rd., Baltimore, MD 21227

Prof. Christopher Farrell, Dept. of Meteorology, 505 Walker Bldg. Pennsylvania State University, University Pk., PA 16802

Dr. Paul Frenzen, Argonne National Laboratory, Building 181, Argonne, IL 60439

Dr. Robert G. Lamb, Jr., Meteorology and Assessment Div., Environmental Protection Agency, Research Triangle Pk., NC 27711

Dr. Gordon A. McBean, Institute of Ocean Sciences, P.O. Box 6000; Sidney, B.C., Canada V8L 4B2

Dr. Richard T. McNider, Chief, Planning and Development, Alabama Air Pollution Control Commission, State Capitol, 645 S. McDonough St., Montgomery, AL 36130

Dr. Fred D. White, National Academy of Sciences, 2101 Constitution Ave. N.W., Washington, DC 20418

Prof. Zellman Warhaft, Sibley School of Mechanical and Aerospace Engineering, 238 Epson Hall, Cornell University, Ithaca, NY 14853

Dr. John C. Wyngaard, National Center for Atmospheric Research, P.O. Box 3000, Boulder, CO 80307

Chairman: Prof. Wayne R. Sand, Dept. of Atmospheric Sciences, University of Wyoming, Laramie, WY 82071

Dr. Harrison E. Cramer, Cramer and Company, P.O. Box 8049, Salt Lake City, UT 84108

\section{Aviation Meteorology}


Biometeorology anc Aerobiology

\section{Climate Variations}

Cloud Physics
Dr. Sepp J. Froeschl, Atmospheric Environment Service, Quebec Forecast Office, 1845 Brookdale, Dorval, Quebec, Canada H9P TX5

Jack W. Hinkelman, Jr., NCAR-PROFS, NOAA/ERL, Department of Commerce, Boulder, CO 80302

Dr. Joseph Kelley, Global Weather Dynamics, Inc., 2400 Garden Rd., Monterey, CA 93940

Dr. Eric Mandel, FAA, Weather Processors Program, APM-640, 800 Independence Ave., S. W., Washington, DC 20591

Howard Mason, Federal Aviation Administration Technical Ctr., ACT-200, Atlantic City Airport, NJ 08405

Dr. John McCarthy, NCAR, P.O. Box 3000, Boulder, CO 80307

Jerald H. Uecker, W/OM 13xl, Aviation Services Br., National Weather Service, NOAA/ Department of Commerce, Gramax Building, 8060 13th St., Silver Spring, MD 20910

John B. Young, Manager Meteorology, Room 235, Hangar 12, JFK International Airport, Jamaica, NY 11430

Lt. Col. Joseph A. Zack, Chief Aerospace Sciences Division, HQ 5th Weather Wing, Langley AFB, VA 23365

Chairman: Prof. Robert L. Edmonds, College of Forestry Resources, University of Washington, Seattle, WA 98195

Dr. Donald E. Aylor, Chief, Dept. of Ecology and Climatology, Connecticut Agricultural Experiment Station, P.O. Box 1106, New Haven, CT 06504

Dr. Larry G. Bergland, John B. Pierce Foundation, 290 Congress Ave., New Haven, CT 06519

Prof. G. Edgar Folk, Jr., Dept. of Physiology and Biophysics, University of Iowa, Iowa City, IA 52242

Dr. G. Leroy Hahn, USDA Meat Animal Research Center, P.O. Box 166, Clay Center, NE 68933

Prof. Conrad J. Mason, Dept. of Atmospheric and Oceanic Sciences, University of Michigan, Ann Arbor, MI 48109

Dr. Lawrence E. Truppi, Terrain Effects Br., Meteorology and Assessment Div., EPA-ESRL, MD-80, Research Triangle Pk., NC 27711

Prof. Kyaw Tha Paw U, Dept. of Agronomy, Purdue University, W. Lafayette, IN 47907

Prof. Chris D. Upper, Dept. of Plant Pathology, University of Wisconsin, Madison, WI 53706

Chairman: Dr. Michael C. MacCracken, Lawrence Livermore Laboratory, University of California, P.O. Box 808, L-262, Livermore, CA 94550

Prof. Raymond S. Bradley, Geology and Physiology Dept., Morrill Science Ctr., University of Massachusetts, Amherst, MA 01003

Dr. Mark A. Cane, Geological Observatory, Lamont-Doherty, Palisades, NY 10964

Dr. Henry F. Diaz, NOAA/ERL Code R/E21, 325 Broadway, Boulder, CO 80303

Dr. Kirby J. Hanson, R/E/ARx2, Dept. of Commerce/ERL/NOAA, 325 Broadway, Boulder, CO 80303

Dr. George J. Kukla, Lamont Observatory, Lamont-Doherty, Palisades, NY 10964

Dr. Kikuro Miyakoda, Geophysical Fluid Dynamics Laboratory, Princeton University, P.O. Box 308, Princeton, NJ 08540

Dr. Gerald R. North, Climate and Radiation Br., Code 915, NASA/Goddard Space Flight Ctr., Greenbelt, MD 20771

Prof. Jagadish Shukla, Dept. of Meteorology, University of Maryland, College Park, MD 20742

Chairman: Dr. David B. Johnson, Illinois State Water Survey, 605 E. Springfield, P.O. Box 5050Station A, Champaign, IL 61820

Dr. D. Ray Booker, President, Aeromet, Inc., P.O. Box 45447, Tulsa, OK 74145

Dr. G. David Emmitt, Simpson Weather Associates, 809 E. Jefferson St., Charlottesville, VA 22902

Dr. Marianne English, Alberta Research Council, Room 715, Terrace Plaza, 4445 Calgary Trail South, Edmonton, Alberta, Canada T6H 5R7 


\section{Hydrology}

\section{Interaction of the Sea and Atmosphere}

Dr. James W. Fitzgerald, Naval Research Laboratory, Code 4326, Washington, DC 20375 Dr. William D. Hall, National Center for Atmospheric Research, P.O. Box 3000, Boulder, CO 80307

Dr. Andrew J. Heymsfield, Convective Storms Division, National Center for Atmospheric Research, P.O. Box 3000, Boulder, CO 80307

Prof. Walter F. Hitschfeld, International Commission on Cloud Physics, IAMAP/IUGG, McGill University, 805 Sherbrooke St. W., Montreal, Quebec, Canada H3A 2K6

Prof. Robert A. Houze, Jr., Dept. of Atmospheric Sciences, AK-40, University of Washington, Seattle, WA 98195

Dr. Thomas J. Matejka, National Center for Atmospheric Research, P.O. Box 3000, Boulder, CO 80307

Chairman: John L. Vogel, Illinois State Water Survey, 605 E. Springfield Ave., P.O. Box 5050, Station A, Champaign, IL 61820

Robert J. C. Burnash, California-Nevada River Forecast Center, NWS, Sacramento, CA 95814

Prof. Peter S. Eagleson, Massachusetts Institute of Technology, Room 48-33S, Cambridge, MA 02139

Bill Hogg, Atmospheric Environment Service, 4905 Dufferin St., Downsview, Ontario, Canada M3H 5T4

Dr. Peter K. Kitanidis, Iowa Institute of Hydraulic Research, University of Iowa, Iowa City, IA 52242

Dr. Alan Lumb, U.S. Geological Survey, 12201 Sunrise Valley Dr., Reston, VA 22091

H. Michael Mogil, 7105 Church Park Dr., P.O. Box 16057, Fort Worth, TX 76133

Dr. Herbert B. Osborne, Southwest Water Research Ctr., 2000 E. Allen Rd., Tucson, AZ 85719

Prof. A. Ramachandra Rao, Dept. of Civil Engineering, Purdue University, West Lafayette, IN 47907

Dr. Vincent V. Salomonson, Chief, Earth Survey Applications Div., NASA/Goddard Space Flight Center, Code 920, Greenbelt, MD 20771

Chairman: Dr. Mark A. Donelan, Shore Processes Section, Hydraulics Div., National Water Research Institute, Canada Centre for Inland Waters, P.O. Box 5050, Burlington, Ontario, Canada LTR YA6

Prof. Roland W. Garwood, Dept. of Oceanography, Code 68 Gd, Naval Postgraduate School, Monterey, CA 93943

Dr. Glenn D. Hamilton, NOAA Data Buoy Center, NOAA/Department of Commerce, Bay St. Louis, MS 39529

Dr. Thomas Hopkins, Dept. of Energy and Environment, Bldg. 318, Brookhaven National Laboratory, Upton, Long Island, NY 11973

Prof. Kristina B. Katsaros, Dept. of Atmospheric Sciences, AK-40, University of Washington, Seattle, WA 98105

Prof. Ruby Krishnamurti, Dept. of Oceanography, Florida State University, Tallahassee, FL 32306

Duncan B. Ross, Jr., NOAA/AOML/SAIL, 15 Rickenbacker Causeway, Miami, FL 33149

Prof. S. SethuRaman, Dept. of Marine, Earth and Atmospheric Sciences, North Carolina State University, Raleigh, NC 27650

Prof. Richard C. J. Somerville, Scripps Institution of Oceanography, A-024, University of California-San Diego, La Jolla, CA 92093

Chairman: Dr. A. I. Carswell, Dept. of Physics and CRESS, York University, 4700 Keele Street, Downsview, Ontario, Canada M3J 1P3

Dr. Edward V. Browell, NASA/Langley Research Center, Mail Stop 401A, Hampton, VA 23665

Dr. Motowa Fujiwara, Dept. of Physics, Kyushu University, Fukuoka, Japan

Dr. R. Milton Huffaker, WOAA/ERL/WPL, Dept. of Commerce, 325 Broadway, Boulder, CO 80303

Dr. Dennis K. Killinger, Lincoln Laboratories, 242 Wood St., Lexington, MA 02173 


\section{Mesoscale Meteorology}

\section{Meteorological Aspects of Air Pollution}

\section{Meteorology of the Coastal Zone}

Dr. Gerard J. Megie, CNRS Service d'Aéronomie, B.P. No. 3, 91370 Verrières le Buisson, France Dr. M. Patrick McCormick, Head, Aerosol, Measurements Research Br., Mail Stop 475, NASA/ Langley Research Center, Hampton, VA 23365

Prof. Kenneth Sassen, Dept. of Meteorology, University of Utah, Salt Lake City, UT 84112 Dr. Lance Thomas, Dept. of Physics, University College of Wales Aberystwyth, Dyfed, United Kingdom

Chairman: Prof. Kerry A. Emanuel, Dept. of Meteorology and Physical Oceanography, Massachusetts Institute of Technology, Cambridge, MA 02139

Prof. Lance F. Bosart, Dept. of Atmospheric Sciences, E.S. 227 State University of New York at Albany, 1400 Washington St., Albany, NY 12222

Prof. J. Michael Fritsch, Dept. of Meteorology, Pennsylvania State University, 503 Walker Bldg., University Park, PA 16802

Prof. Christopher J. R. Garrett, Dept. of Oceanography, Dalhousie University, Halifax, Nova Scotia, Canada B3H $4 \mathrm{~J} 1$

Dr. Daniel Keyser, NASA/Goddard Space Flight Center, Code 914, Greenbelt, MD 20771

Prof. Douglas K. Lilly, Dept. of Meteorology, University of Oklahoma, 200 Felgar St., Norman, OK 73019

Dr. Robert A. Maddox, Weather Research Program-R/E22, NOAA/ERL/WRP, 325 Broadway, Boulder, CO 80303

Dr. Isidoro Orlanski, Geophysical Fluid Dynamics Laboratory, NOAA/Department of Commerce, Forrestal Campus, Princeton University, P.O. Box 308, Princeton, NJ 08542

Dr. Edward J. Zipser, National Center for Atmospheric Research, P.O. Box 3000, Boulder, CO 80307

Chairman: Dr. Carmen J. Nappo, Jr., Research Meteorologist, Department of Commerce/ NOAA, Oak Ridge National Laboratory, Oak Ridge, TN 37830

Dr. Gordon A. Beals, Consolidated Edison Co. of New York, Rm. 306S, 4 Irving Place, New York, NY 10003

Dr. John F. Clarke, EPA Meteorological Laboratory, Research Triangle Pk., NC 27711

Dr. Raymond J. DeLand, Ray DeLand and Assoc., Ltd., 2 Collyer Dr., Ossining, NY 10562

Paul M. Fransioli, Black and Veatch Consulting Engineers, P.O. Box 8405, Kansas City, MO 64114

Dr. Ramesh V. Godbole, Senior Environmental Engineer, Air Quality Dept., Potomac Electric Power Co., 1900 Pennsylvania Ave. N.W., Washington, DC 20068

Dr. William M. Porch, Atmospheric and Geophysic Science Div., Lawrence Livermore Laboratory, P.O. Box 808, Livermore, CA 94550

Dr. Darryl Randerson, NOAA, P.O. Box 14985, Las Vegas, NV 89114

Prof. John D. Spengler, Dept. of Environmental Health Sciences, Harvard University, 655 Huntington Ave., Boston, MA 02115

Dr. Akula Venkatram, ERT, 696 Virginia Rd., Concord, MA 01742

Chairman: Robert W. Fett, Director of Tactical Applications, Naval Environmental Prediction Research Facility, Monterey, CA 93940

John A. Bujnoch, Jr., Conoco, Inc., Rm. 2704, P.O. Box 2197, Houston, TX 77001

Prof. Mariano A. Estoque, RSMAS/MPO, University of Miami, 4600 Rickenbacker Causeway, Miami, FL 33149

Prof. Bruce P. Hayden, Dept. of Environmental Sciences, University of Virginia, Charlottesville, VA 22903

Prof. S. A. Hsu, Coastal Studies Institute, Louisiana State University, Baton Rouge, LA 70803 Thomas H. Kinder, Physical Oceanography Branch, Naval Ocean Research \& Development Activity, NSTL, MS 39529

Marvin E. Miller, National Weather Service, NOAA, Federal Facilities Bldg., Cleveland-Hopkins Airport, Cleveland, $\mathrm{OH} 44135$ 
Meteorology of the Southern Hemisphere

\section{Middle Atmosphere}

\section{Mountain Meteorology}

Robert G. Quayle, National Climatic Data Center, Federal Bldg., Asheville, NC 28801

Dr. Omar H. Shemdin, Jet Propulsion Laboratory, 4800 Oak Grove Dr., Mail Stop 183-501, Pasadena, CA 91109

Dr. Robert H. Simpson, Simpson Weather Associates, 6200 Westchester Pk., Apt. 517, College Park, MD 20740

Chairman: Prof. Julia N. Paegle, Dept. of Meteorology, University of Utah, 819 William C. Browning Bldg., Salt Lake City, UT 84112

Dr. George C. Cressman, National Meteorological Center, World Weather Bldg., Rm. 204, 5200 Auth Rd., Washington, DC 20233

Dr. Douglas J. Gauntlett, ANMRC, Box 5089, Melbourne, Australia 3001

Dr. Eugenia Kalnay, NASA/Goddard Space Flight Center, Code 911, Greenbelt, MD 20771

Dr. Gustavo Necco, World Weather Bldg., 5200 Auth Rd., Washington, DC 20233

Prof. Kevin E. Trenberth, National Center for Atmospheric Research, P.O. Box 3000, Boulder, CO 80307

Dr. Harry Van Loon, National Center for Atmospheric Research, P.O. Box 3000, Boulder, CO 80307

Prof. Dayton G. Vincent, Dept. of Geosciences, Purdue University, West Lafayette, IN 47907 Dr. Peter J. Webster, Dept. of Meteorology, Pennsylvania State University, University Park, PA 16801

Chairman: Dr. Murray L. Salby, Department of Astrophysical, Planetary, and Atmospheric Sciences, University of Colorado, Campus Box 391, Boulder, CO 80309

Dr. Ben B. Balsley, Aeronomy Laboratory, ERL/NOAA, 325 Broadway-R/E/AL3, Boulder, CO 80303

Prof. John P. Boyd, Dept. of Atmospheric and Oceanic Sciences, University of Michigan, 2455 Hayward, Ann Arbor, MI 48109

Dr. Jeffrey M. Forbes, Dept. of Physics, Boston College, Chestnut Hill, MA 02167

Dr. Marvin A. Geller, Code 964, NASA/Goddard Space Flight Center, Greenbelt, MD 20771

Prof. James R. Holton, Dept. of Atmospheric Sciences, AK-40, University of Washington, Seattle, WA 98195

Dr. Keith W. Johnson, Climate Analysis Center, NWS/NOAA, Washington, DC 20233

Prof. Alan H. Manson, Physics Dept., University of Saskatchewan, Saskatoon, Saskatchewan, Canada S7N 0W0

Dr. James M. Russell, III, Mail Stop 401A, NASA/Langley Research Center, Hampton, VA 23665

Dr. Mark R. Schoeberl, NASA/Goddard Space Flight Center, Code 964, Greenbelt, MD 20771

Dr. Susan Solomon, Aeronomy Laboratory, Dept. of Commerce/NOAA/ERL, 325 Broadway, Boulder, CO 80303

Dr. Darrell F. Strobel, Plasma Physics Div., Code 4780, Naval Research Laboratory, Washington, DC 20375

Chairman: Douglas G. Fox, Chief Meteorologist, U.S. Department of Agriculture Forest Service, Rocky Mountain Forest and Range Experiment Station, 240 W. Prospect St., Ft. Collins, CO 80526

Dr. William E. Clements, Project Leader for Meteorological Physics, Los Alamos National Laboratory, P.O. Box 1663, MS-466, Los Alamos, NM 87544

Loren W. Crow, Loren W. Crow Consultants, Inc., 3064 South Monroe St., Denver, CO 80210

Dr. Donna V. Lamb, U.S. Forest Service, 319 S. W. Pine St., P.O. Box 3623, Portland, OR 97204

Prof. William E. Marlatt, Chairman/Environmental Sciences, Dept. of Engineering Ecology, Colorado School of Mines, Golden, CO 80401

Prof. Elmar R. Reiter, C.S.U. Solar House \#3, Ft. Collins, CO 80523

Prof. Ronald B. Smith, Dept. of Geology and Geophysics, Yale University, New Haven, CT 06520 


\section{Planned and Inadvertent Weather Modification}

Dr. William T. Sommers, Forest Fire and Atmospheric Sciences Research Staff, USDA, Forest Service, P.O. Box 2417, Washington, DC 20013

Prof. Gene L. Wooldridge, Dept. of Soil Sciences and Biometeorology, Utah State University, Logan, UT 84322

Chairman: Stanley A. Changnon, Chief, Illinois State Water Survey, 605 E. Springfield, Box 5050-Station A, Champaign, IL 61820

Prof. Roscoe R. Braham, Jr., Dept. of the Geophysical Sciences, University of Chicago, 5734 S. Ellis Ave., Chicago, IL 60637

Keith J. Brown, North American Weather Consultants, 1141 East 3900 South A 130, Salt Lake City, UT 84117

Dr. Arnett S. Dennis, Denver Federal Center, P.O. Box 25007, Mail Code D-1210, Denver, CO 80225

Dr. Richard A. Dirks, Director of GALE Project Office, NCAR/GALE Project Office, P.O. Box 3000, Boulder, CO 80307

Dr. G. Brant Foote, National Center for Atmospheric Research, P.O. Box 3000, Boulder, CO 80307

Dr. Edward E. Hindman, II, Department of Atmospheric Sciences, Colorado State University, Fort Collins, CO 80523

Dr. Robert G. Humphries, Alberta Research Council, Industrial and Engineering Research Div., 4th Floor Terrace Plaza, 4445 Calgary Trail South, Edmonton, Alberta, Canada T6H 5R7

Prof. Harold D. Orville, Head, Dept. of Meteorology, South Dakota School of Mines and Technology, Rapid City, SD 57701

Prof. Joseph A. Warburton, Desert Research Institute, University of Nevada, Reno, NV 89507

Chairman: Prof. John E. Walsh, Jr., Laboratory for Atmospheric Research, University of Illinois, 1101 W. Springfield Ave., Urbana, IL 61801

Stephen F. Ackley, U.S. Army CRREL, Box 282, Hanover, NH 03755

Prof. Austin W. Hogan, Earth Science Bldg., Rm. 324, State University of New York, 1400 Washington Ave., Albany, NY 12222

Prof. Kolf D. Jayaweera, Geophysical Institute, University of Alaska, Fairbanks, AK 99701

Prof. Seelye Martin, Dept. of Oceanography, University of Washington, WB-10, Seattle, WA 98195

Dr. J. Murray Mitchell, NOAA/NESDIS, 8th Floor, WSC 5, Code R/E/AR, 6010 Executive Blvd., Rockville, MD 20852

Dr. Claire L. Parkinson, Oceans and Ice Br., Code 912, NASA/Goddard Space Flight Center, Greenbelt, MD 20771

Prof. Elmer Robinson, College of Engineering, Air Pollution Section, Washington State University, Pullman, WA 99164

Dr. Gary R. Schram, Officer in Charge, Arctic Weather Centre, Atmospheric Environment Service, Environment Canada, 6325 103rd St., Edmonton, Alberta, Canada T6H 5H6

Chairman: Dr. Robert E. Livezey, Climate Analysis Ctr., National Weather Service/NOAA, World Weather Bldg., Washington, DC 20233

Dr. Robert F. Cahalan, Code 915, NASA/Goddard Space Flight Center, Greenbelt, MD 20771

Dr. Paul R. Julian, National Center for Atmospheric Research, P.O. Box 3000, Boulder, CO 80307

Dr. Richard W. Katz, National Center for Atmospheric Research, P.O. Box 3000, Boulder, CO 80307

Prof. Paul W. Mielke, Jr., Colorado State University, Dept. of Statistics, Fort Collins, CO 80523 Dr. Robert Miller, Techniques Development Laboratory, U.S. Dept. of Commerce/NOAA/ NWS, 8060 13th St., Silver Spring, MD 20910

Michael B. Richman, Illinois State Water Survey, P.O. Box 5050, Station A, Champaign, IL 61820 
Radar Meteorology

\section{Radiation Energy}

\section{Satellite and Aerospace Systems}

G. Louis Smith, Mail Stop 420, NASA/Langley Research Ctr., Hampton, VA 23665

Prof. Grace Wahba, Dept. of Statistics, University of Wisconsin, 1210 W. Dayton Street, Madison, WI 53706

Dr. Nathan Yacowar, Head, Techniques Development Section, Canadian Meteorological Centre, 2121 North Service Rd., Suite 404, Dorval, Quebec, Canada H9P 1J3

Chairman: Dr. Peter S. Ray, National Severe Storms Laboratory, NOAA, ERL/R/E/NS, 1313 Halley Circle, Norman, OK 73069-8493

Dr. David Atlas, Chief, Laboratory for Atmospheric Sciences, NASA/Goddard Space Flight Center, Greenbelt, MD 20771

Richard E. Carbone, National Center for Atmospheric Research, P.O. Box 3000, Boulder, CO 80307

Dr. Christopher G. Collier, Meteorological Office Radar Research Laboratory, BY Bldg., Royal Signals and Radar Establishment, St. Andrews Rd., Great Malvern, Worcestershire, WR14 3PX, England

Dr. Arthur R. Jameson, State Water Survey Division, Illinois Dept. of Energy and Natural Resources, 605 East Springfield Ave., P.O. Box 5050, Station A, Champaign, IL 61820

Dr. Fausto Pasqualucci, NOAA/ERL/WPL, R/E/WP6, 325 Broadway, Boulder, CO 80303 Prof. Richard E. Passarelli, Jr., Dept. of Meteorology and Physical Oceanography, Rm. 541818, Massachusetts Institute of Technology, Cambridge, MA 02139

Prof. Roddy R. Rogers, Meteorology Dept., McGill University, 805 Sherbrooke St. W, Montreal, Quebec, Canada H3A 2K6

Robert J. Serafin, Director, Atmospheric Technology Div., National Center for Atmospheric Research, P.O. Box 3000, Boulder, CO 80307

Dr. Albert Waldvogel, Atmospheric Physics Laboratory, Eidgenossische Technische Hochschule, Honggerberg, Zurich, Switzerland HPP-8093

Chairman: Dr. Robert J. Curran, NASA HQ, Code EE-8, Washington, DC 20546

Dr. Thomas P. Ackerman, Research Scientist, Theoretical and Planetary Studies Br. SST-245-3, Space Science Div., NASA/Ames Research Center, Moffett Field, CA 94035

Prof. Bruce A. Albrecht, Dept. of Meteorology, Pennsylvania State University, University Pk., PA 16801

Dr. Bruce Barkstrom, Head, ERB Project, NASA/Langley Research Center, Code 423, Hampton, VA 23665

Dr. John DeLuisi, Geophysical Monitoring for Climate Change, ERL/NOAA, R/E/AR4, Boulder, CO 80303

Dr. Lewis D. Kaplan, Atmospheric and Environmental Research, Inc., 840 Memorial Drive, Cambridge, MA 02139

Dr. Michael D. King, Climate and Radiation Br., Code 915, NASA/Goddard Space Flight Center, Greenbelt, MD 20771

Prof. Kuo-Nan Liou, Dept. of Meteorology, University of Utah, Salt Lake City, UT 84112 Prof. Rachel Pinker, Dept. of Meteorology, University of Maryland, College Pk., MD 20742 Dr. V. Ramanathan, Climate Project, National Center for Atmospheric Research, P.O. Box 3000, Boulder, CO 80307

Chairman: Prof. Thomas H. Vonder Haar, Dept. of Atmospheric Sciences, Colorado State University, Fort Collins, CO 80523

Dr. George H. Fichtl, Chief, Fluid Dynamics Br., Atmospheric Science Div., NASA/Marshall Space Flight Center, Code ED42, Huntsville, AL 35812

Prof. Walter Frost, Director, Atmospheric Sciences Div., University of Tennessee Space Institute, Tullahoma, TN 37388

Col. Frank Globaker, Air Force Systems Command Space Div., Code SD/WE, P.O. Box 92960, Worldway Postal Center, Los Angeles, CA 90000

Dr. Kenneth R. Hardy, Air Force Geophysical Laboratory, Satellite Meteorology Br., AFGL/ LYS, Hanscom AFB, MA 01731 
Dr. Arthur F. Hasler, NASA/Goddard Space Flight Center, Code 914, Greenbelt, MD 20771 Dr. Stanley Q. Kidder, State Water Survey Division/ENR, Water Resources Bldg., P.O. Box 5050, 605 E. Springfield, Champaign, IL 61820

Dr. Eric Mollo-Christensen, Head, Ocean and Ice Br., Goddard Laboratory for Atmospheric Sciences, Code 912, NASA/Goddard Space Flight Center, Greenbelt, MD 20771

Thomas H. Pries, U.S. Army Atmospheric Sciences Laboratory, ATTN: DELAS-AT, White Sands Missile Range, NM 88002

Dr. William L. Smith, Dept. of Commerce/NOAA, Satellite Section, Space Science and Engineering Ctr., University of Wisconsin, 1225 W. Dayton St., Madison, WI 53706

John S. Theon, Acting Chief, Atmospheric Dynamics and Radiation Br., NASA HQ, EE-8, Washington, DC 20546

Dr. William W. Vaughan, Chief, Atmospheric Sciences Div., ES-41, NASA/Marshall Space Flight Center, Huntsville, AL 35812

\section{Severe Local Storms}

Tropical Meteorology and Tropical Cyclones
Chairman: Dr. Ralph A. Petersen, Code 914, NASA/Goddard Space Flight Center, Greenbelt, MD 20771

Dr. Ronald L. Alberty, NOAA/PROFS Office RX6, 325 Broadway, Boulder, CO 80303

Prof. Howard B. Bluestein, School of Meteorology, University of Oklahoma, 200 Felgar Street, Norman, OK 73019

Dr. Donald W. Burgess, National Severe Storms Laboratory, 1313 Halley Circle, Norman, OK 73069

Dennis W. Feltgen, KSTP Weather Services, 3415 University Ave., St. Paul, MN 55414

Prof. J. Michael Fritsch, Pennsylvania State University, Dept. of Meteorology, 503 Walker Bldg., University Pk., PA 16802

Dennis H. McCarthy, NWS/NOAA, 4100 Mexico Rd., St. Charles, MO 63301

Dr. Richard Rotunno, NCAR, P.O. Box 3000, Boulder, CO 80307

Prof. Thomas A. Schroeder, Dept. of Meteorology, University of Hawaii at Manoa, 2525 Correa Rd., Honolulu, HI 96822

Prof. John T. Snow, Dept. of Geosciences, Purdue University, West Lafayette, IN 47907

Chairman: Dr. Stanley L. Rosenthal, Deputy Director, (Atmospheric Programs, AOML), Hurricane Research Division, AOML, ERL, NOAA, Department of Commerce, 4301 Rickenbacker Causeway, Miami, FL 33149

Dr. Alan K. Betts, Rural Delivery, West Pawlet, VT 05775

Prof. Russell L. Elsberry, Dept. of Meteorology, Naval Postgraduate School, Monterey, CA 93940

Prof. William M. Frank, Dept. of Meteorology, Pennsylvania State University, University Pk., PA 16802

Dr. John C. Freeman, Institute for Storm Research, 3600 Mount Vernon St., Houston, TX 77006

Prof. T. N. Krishnamurti, Dept. of Meteorology, Florida State University, Tallahassee, FL 32306

Prof. Colleen A. Leary, Atmospheric Science Group, Texas Tech University, Box 4320, Lubbock, TX 79409

Dr. Margaret A. LeMone, National Center for Atmospheric Research, P.O. Box 3000, Boulder, CO 80307

Dr. Charles J. Neumann, National Hurricane Center, National Weather Service, NOAA/Department of Commerce, Gables One Tower, 1320 S. Dixie Highway, Coral Gables, FL 33146

Dr. Herbert Riehl, CIRES, 4390 Caddo Parkway, Boulder, CO 80303

Chairman: Dr. Alan I. Weinstein, Leader, Ocean Sciences Div., Office of Naval Research, Code 422, 800 N. Quincy St., Arlington, VA 22217

Robert G. Behm, Deputy-MIC, National Weather Service Forecast Office-Phoenix, 2633 E. Buckeye Rd., Phoenix, AZ 85034 
Dr. Donald W. Beran, Chief, ERL/NOAA/WPL, R/E/WP4 Remote Profiling, 325 Broadway, Boulder, CO 80303

Gilbert B. Clark, National Hurricane Center, National Weather Service, NOAA/Department of Commerce, Gables One Tower, 1320 S. Dixie Highway, Coral Gables, FL 33146

Dr. Roger W. Daley, National Center for Atmospheric Research, P.O. Box 3000, Boulder, CO 80307-3000

Dr. James E. Hoke, National Meteorological Center, W/NMC22, Rm. 204/WWB, Washington, DC 20233

Colonel James Kerlin, Commander, 11th Weather Sqd., Elmendorf AFB, AK 99506

Robert E. Muller, Deputy MIC, National Weather Service Forecast Office-Raleigh-Durham Airport, P.O. Box 165, Morrisville, NC 27560

Prof. Richard J. Reed, Dept. of Atmospheric Sciences, AK-40, University of Washington, Seattle, WA 98195

Dr. Ian D. Rutherford, Atmospheric Environment Service, 4905 Dufferin St., Downsview, Ontario, Canada M3H 5T4

David B. Spiegler, MITRE Corp., Burlington Road, Bedford, MA 01730

Dr. Louis W. Uccellini, NASA/Goddard Space Flight Center, Code 914, Greenbelt, MD 20771

\section{EDUCATION AND MANPOWER COMMISSION}

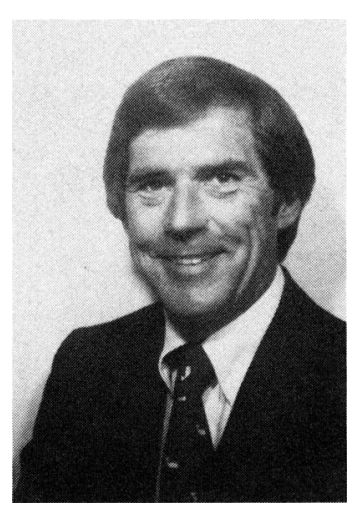

O. E. Thompson

\section{Board of Meteorological and Oceanographic Education in Universities}

Chairman: Prof. Owen E. Thompson, Dept. of Meteorology, University of Maryland, College Park, MD 20742

Members: Chairmen of the constituent Boards

Chairman: Prof. David D. Houghton, Dept. of Meteorology, University of Wisconsin-Madison, Madison, WI 53706

Fred V. Brock, National Center for Atmospheric Research, P.O. Box 3000, Boulder, CO 80307 Prof. David L. Evans, Graduate School of Oceanography, University of Rhode Island, Kingston, RI 02881

Prof. Ira W. Geer, Dept. of Earth Sciences, State University of New York at Brockport, Brockport, NY 14420

Dr. Arnold Gruber, National Environmental and Satellite Data Information Service, NOAA, World Weather Bldg., Suitland, MD 20233

Prof. George J. Huffman, Dept. of Meteorology, University of Maryland, College Park, MD 20742

Prof. Kristina B. Katsaros, Dept. of Atmospheric Sciences, University of Washington, Seattle, WA 98195

Prof. James F. Kimpel, School of Meteorology, University of Oklahoma, Norman, OK 73019

Dr. Lonzy J. Lewis, Dept. of Physics and Atmospheric Science, Jackson State University, Jackson, MS 39217

Frederick P. Ostby, Director, National Severe Storms Forecast Center, NOAA, 601 E. 12th St., Kansas City, MO 64106

Prof. Perry J. Samson, Dept. of Atmospheric and Oceanic Sciences, University of Michigan, Ann Arbor, MI 48109 


\section{Board of School and Popular Meteorological and Oceanographic Education}

\author{
Committee of Judges \\ for Undergraduate \\ Awards
}

\section{Board on Women and Minorities}

Irwin Spickler, 20433 Meadow Pond Pl., Gaithersburg, MD 20879

Prof. Eugene S. Takle, Dept. of Earth Sciences, Iowa State University, Ames, IA 50010

Prof. Andrew C. Vastano, Dept. of Oceanography, Texas A\&M University, College Station, TX 77843

Chairman: Howard A. Friedman, Hurricane Research Div., Atlantic Oceanic and Meteorological Laboratories, NOAA, 4301 Rickenbacker Causeway, Miami, FL 33149

Herbert S. Lieb, 13115 Bluhill Rd., Silver Spring, MD 20906

H. Michael Mogil, 7105 Church Park Dr., Ft. Worth, TX 76133

Prof. Victor T. Neal, School of Oceanography, Oregon State University, Corvallis, OR 97331

John F. Sanders, III, Science Applications, Inc., 205 Montecito Ave., Monterey, CA 93940

Jack Williams, USA TODAY, Box 500, Washington, DC 20044

Chairman: Dr. Sharon K. LeDuc, Center for Environmental Assessment Services, Environmental Data and Information Service, NOAA, 200 Federal Bldg., 600 Cherry St., Columbia, MO 65201 Prof. John J. Cahir, Dept. of Meteorology, Naval Postgraduate School, Monterey, CA 93940 Prof. Dennis L. Hartmann, Dept. of Atmospheric Sciences, University of Washington, Seattle, WA 98195

Chairman: Pamela L. Stephens, Global Atmospheric Research Program, National Science Foundation, 1800 G St., N.W., Washington, DC 20550

Beverly J. Barnhart, Science Applications, Inc., 1710 Goodridge Dr., McLean, VA 22102

Kerry H. Cook, Institute for Energy Analysis, Box 117, Oak Ridge, TN 37830

Dr. Pieter J. Feteris, E. Just Science Hall, Jackson State University, Jackson, MS 39217

Carlos Garza, National Weather Service, NOAA, 1001 International Blvd., Hapeville, GA 30354

Prof. Gerald W. Grams, School of Geophysical Sciences, Georgia Institute of Technology, Atlanta, GA 30332

Lt. Col. Walter J. Harrison, USAF, 302 Inner Circle, Maxwell AFB, AL 36113

Carolyn Hodge, Office of Equal Employment Opportunity, NOAA, World Weather Bldg., Washington, DC 20233

Dr. Sharon E. Nicholson, Dept. of Geography, Clark University, Worcester, MA 01610

Steven J. Richards, 2344 Boston Rd., Bronx, NY 10467

Lesley F. Tarleton, R\&D Consultants, Crestmoor Dr., Boulder, CO 80303

\section{PLANNING COMMISSION}

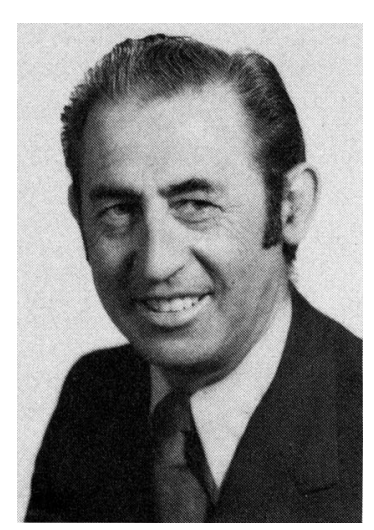

L. J. Battan
Chairman: Dr. Louis J. Battan, Institute of Atmospheric Physics, University of Arizona, Tucson, AZ 85721

Dr. Earl G. Droessler, Assistant Secretary for Natural Resources, North Carolina Dept. of Natural Resources and Community Development, P.O. Box 27687, Raleigh, NC 27611

Dr. Richard E. Hallgren, Assistant Administrator for Weather Services, National Oceanic and Atmospheric Administration, 8060 13th St., Silver Spring, MD 20910

Prof. Charles L. Hosler, Jr., Dean, College of Earth and Mineral Sciences, Pennsylvania State University, University Park, PA 16802

Dr. James R. Mahoney, Manager, Environmental Sciences Dept., Bechtel Corporation, P.O. Box 3965, San Francisco, CA 94119

Prof. Owen M. Phillips, Dept. of Earth and Planetary Sciences, Johns Hopkins University, Baltimore, MD 21218

Prof. Verner E. Suomi, Director, Space Science and Engineering Center, University of Wisconsin-Madison, Madison, WI 53706 